\title{
Progesterone and estradiol may be beneficial for the treatment of COVID-19 (HYPOTHESIS PAPER)
}

\author{
Graziano Pinna ${ }^{1}$ \\ ${ }^{1}$ University of Illinois at Chicago
}

May 11, 2020

\begin{abstract}
SARS-CoV-2 has claimed more than 300,000 lives while infecting 4 million individuals worldwide. There are no specific treatments for COVID-19. Symptoms vary from very mild/asymptomatic to severe, including admission to ICU. Strong sexbias in COVID-19 have been noted with males showing more than double the odds of requiring ICU admission and higher mortality. Reproductive steroids, including estrogens, progesterone and its physiologically-active metabolite, allopregnanolone exert anti-inflammatory actions and influence the immune system. Intriguingly, pregnant women with COVID-19 appear to experience milder symptoms. In some pregnant women escalated symptoms severity is observed immediately postpartum in coincidence with the rapid hormonal drop associated with parturition. This finding suggests that reproductive steroids' antiinflammatory effects and their role in reshaping competence of immune cells may protect during pregnancy. The hypothesis that estradiol, progesterone may provide a treatment against COVID-19 in men and in postmenopausal women is discussed.
\end{abstract}

\section{Introduction}

SARS-CoV-2 is a rapidly spreading pandemic that was first reported on December 31, 2019 in Wuhan, China. As of May $8^{\text {th }}, 2020$ more than 4 million individuals were tested positive and more than 300,000 have died worldwide. Mortality is believed to account for $2 \%$ of patients (1). At risk individuals, include age above 60 years old, pre-existing conditions, such cardiovascular disease, diabetes and hypertension. Furthermore, there is strong sex-bias in COVID-19 with male patients showing more than double the odds of requiring intensive care unit (ICU) admission (OR 2.5) and higher mortality (OR 1.60) when compared to females (2). Symptoms include sore throats, fever, cough, myalgias, chest pain, headache, pneumonia and shortness of breath (3). Severe cases require hospitalization in intensive care units, which is already challenging the healthcare capacity in the US. There is no specific or efficient treatment available, however the US Food and Drug Administration (FDA) has approved the antiviral medicine remdesivir following a positive clinical trial showing faster recovery.

Several case reports have observed that pregnant women with absence of COVID-19 symptoms on admission to obstetrical service escalated symptoms severity immediately postpartum, which in few cases required unplanned ICU admission. There is a general consensus that most pregnant women with COVID-19 appear to experience a milder clinical course (7-9).

The levels of ovarian steroids, including estrogens and progesterone steadily increase during pregnancy before undergoing an abrupt decrease postpartum that reaches basal levels days after delivery (10-12). In 1 each 9 women, this is associated with postpartum psychopathology and sustained inflammation (13). Estrogens, progesterone and its metabolite, allopregnanolone are anti-inflammatory and allopregnanolone is a psychotropic agent that was recently approved for the treatment of postpartum depression $(13,14)$. Hereinafter, the role of reproductive steroids, including estradiol, progesterone and allopregnanolone, as possible protectant against COVID-19 is discussed. 


\section{Levels of progesterone and allopregnanolone during pregnancy and postpartum}

Estradiol, progesterone and allopregnanolone are endogenous steroids, which are abundantly produced in the periphery by adrenal glands and ovaries and by the brain de novo from cholesterol (14). These neuroactive steroids play important neurophysiological roles by modulating inflammatory processes and behavior. Estradiol and progesterone exerts important peripheral and neuronal functions mediated by genomic influencing nuclear receptor. Allopregnanolone, instead, acts as a positive allosteric modulator of the action of GABA at $\mathrm{GABA}_{\mathrm{A}}$ receptors (15), which has been recently associated with rapid and long-lasting improvement of postpartum depression (16).

Circulating levels of estradiol, progesterone and allopregnanolone rise drastically during the second and third trimester of pregnancy before decreasing dramatically following childbirth (10-12). Estradiol and allopregnanolone levels closely parallel progesterone level increase during pregnancy and in the early postpartum period (10-12). This hormonal progression during pregnancy is consistent with a physiological role as hormonal modifiers in pregnancy and postpartum pathophysiological processes (Figure 1A, B) (12-15).

In healthy pregnant women, serum allopregnanolone, estradiol and progesterone levels increased significantly during pregnancy, reaching the highest levels at term (11). In another study, levels of progesterone were markedly elevated specifically from the second to the third trimester. They reached a 100-fold higher concentration compared to baseline at 36.6 weeks of pregnancy. Concentration of allopregnanolone increased from the first to second trimester, and then remained relatively elevated until after delivery. Postpartum concentrations of both progesterone and allopregnanolone decreased sharply to pre-pregnancy baseline levels (11-13).

Peripartum concentrations of estradiol, progesterone and allopregnanolone have consistently been associated with behaviorally alterations in the spectrum of mood swings and postpartum depression, and increased inflammation in more than $10 \%$ of pregnant women (13).

Progesterone and estrogen effects on inflammation and role in shaping immune cell competence

In addition to the well-known role of progesterone in reproduction, this steroid regulates important immunomodulatory functions, including reshaping the competence of immune cells and inducing potent antiinflammatory actions. Progesterone plays a significant role in the maternal reproductive apparatus, such as immune adaptations and inducing immune tolerance that promote and sustain pregnancy.

There are several ways by which progesterone induce immunomodulatory effects (Figure 1C). These include actions in the periphery where progesterone has an effect on $\mathrm{T}$ cell activation and a direct role on guiding their differentiation. For example, progesterone can modulate $\mathrm{T}$ cell receptor signal transduction or induce tolerance. Progesterone plays also a significant role in suppression of cellular cytotoxicity. Indeed, it may block degranulation by influencing via progesterone induced blocking factor.

Intriguingly, progesterone binds to receptors in immune cells, such as natural killer cells, T cells, macrophages, and dendritic cells. It also binds non-immune cells, including epithelial and endothelial cells in the respiratory tracts where it alters cellular signaling/activity improving infections. In influenza A virus, preclinical progesterone administration decreases inflammation and promotes pulmonary repair after clearance of influenza A viruses by increasing regulatory CD39+ Th17 cells and stimulating TGF-b, IL-6, and IL-22 levels. IL-22 similarly to TGF- $\beta$ can stimulate the proliferation of epithelial cells and promote repair of the damaged alveolar epithelium. Progesterone signaling through progesterone receptors stimulates the epidermal growth factor amphiregulin thereby promoting proliferation and respiratory epithelial cell repair (31). A faster recovery of the lung tissue may reduce in females the susceptibility to secondary bacterial infection. In humans, this is the primary cause of mortality after influenza A virus infection (31). This finding is substantiated by studies showing that following influenza A virus infection, the histological density of pulmonary inflammation was decreased during pregnancy. These investigators conclude that "pregnancy-associated alterations in pulmonary physiology may serve to protect females during severe influenza." (32). Combination of estradiol with progesterone showed the strongest protective role in the lungs after the stimulation of the inflammatory 
cascade thereby explain a milder clinical outcome that has been observed in virus infected women in early compared with late gestation (33).

Like progesterone, estrogens are strong immune regulatory agent. Estrogens regulate immune cell responses and promote anti-inflammatory and neuroprotective effects. Increase in circulating estrogen concentration affects progenitor and mature cells of both the innate and adaptive immune systems (34).

In the innate immune system estrogens regulate the number of cells and their functions. In neutrophils, estrogens regulate chemotaxis, infiltration, as well as the induction of cytokine-induced chemoattractants and cytokines (e.g., TNF- $\alpha$, IL-6, IL-1 $\beta$ ). In dendritic cells, they stimulate the differentiation and directly regulate expression of chemokine (e.g., IL-8) and cytokine (e.g., IL-6, IL-10). In macrophages, estrogens regulate chemotaxis, phagocytic activity, and the production of cytokines (e.g., IL-6, TNF- $\alpha$ ). Estrogens influence the phenotype of $\mathrm{T}$ helper cells and have profound effects on $\mathrm{B}$ cell maturation, differentiation, activity, and survival (34). Estradiol modulates cytokine secretion by CD4+ T cells. Intriguingly, during menopause women are at higher risk of autoimmune diseases. This decline in the content of estrogens during menopause reduces the number of $\mathrm{B}$ and $\mathrm{T}$ cells while increasing production of pro-inflammatory cytokines (36).

\section{Anti-inflammatory effects of allopregnanolone are mediated by $\mathrm{GABA}_{\mathrm{A}}$ receptors}

GABA-active agents possess pleitropic actions and are prescribed to treat anxiety, epilepsy, alcohol use disorder and induce sedation and anesthesia (11). Immune cells express receptors for several neurotransmitters, neuropeptides, and hormones. Of note, the GABAergic system can be modulated endogenously within the immune system (12). GABA possesses important anti-inflammatory actions and GABA $\mathrm{A}_{\mathrm{A}}$ receptors are widely expressed in immune competent cells (13). Indeed, treatment with GABA downregulates autoimmunity mediated by T-cells, inflammation and ameliorates cytotoxic immune responses (14). Activation of $\mathrm{GABA}_{\mathrm{A}}$ receptors by GABA or their agonists decreased pro-inflammatory cytokines in peripheral macrophages. GABAergic agents also protect in models of experimental autoimmune encephalomyelitis, which underscores the role of $\mathrm{GABA}_{\mathrm{A}}$ receptors within the immune system. In support, GABA secretion by both antigen presenting cells (APCs) and $\mathrm{T}$ cells, functional $\mathrm{GABA}_{\mathrm{A}}$ receptors on macrophages, and a direct effect of GABA-active molecules on APCs were reported (14). GABA $A_{A}$ receptor subtypes were identified in human, mouse and rat $\mathrm{CD} 4+$ and $\mathrm{CD} 8+\mathrm{T}$ cells. Abundant $\mathrm{GABA}_{\mathrm{A}}$ receptors were observed in $\mathrm{T}$ cells from human and rodents with functional $\mathrm{GABA}_{\mathrm{A}}$ channels expressing a variety of subunit subtypes $(15,16)$.

In macrophages, the toll-like receptor 4 (TLR4)-specific ligand, lipopolysaccharide (LPS), induces proinflammatory cytokines and chemokines (17). Peripheral inflammation also signals to the brain with production of pro-inflammatory responses. TLR4-mediated pathway is also expressed in neurons. The TLR4 signal cascade involves activation of the $\mathrm{GABA}_{\mathrm{A}}$ receptor-containing $\alpha 2$ subunit $(17,18)$.

Allopregnanolone shows protective functions in preclinical studies, including depression, schizophrenia, traumatic brain injury, alcohol use disorder, multiple sclerosis, Parkinson's and Alzheimer's disease (17). These pathophysiological conditions enhance proinflammatory signaling mediated by TLR activation in peripheral organs and brain (Figure 1B). Monocytes/macrophages were studied as a model of peripheral immune signaling activation (18) showing LPS activated the TLR4 pathway with downstream activation of NFkB and increased pro-inflammatory mediators (MCP-1 and TNF- $\alpha$ ). Allopregnanolone, by inhibiting TLR4 signaling, consistently blocked pro-inflammatory marks with a mechanism that involved blockade of TLR4/MD-2 protein interactions in macrophages (18). This effect was also demonstrated in brain (18). Hence, inhibition of pro-inflammatory processes both in the peripheral and neuroimmune signaling, which can be initiated by allopregnanolone binding at $\mathrm{GABA}_{\mathrm{A}}$ receptor in monocytes/macrophages and brain cells, underscores the profound protective effects of allopregnanolone. This anti-inflammatory response by allopregnanolone inhibition of TLR4 signal underlies a novel function of allopregnanolone to regulate periphery and the brain immune responses (18).

Estrogens, progesterone and allopregnanolone may protect against Covid-19 during pregnancy 
The finding that in Covid-19-positive asymptomatic pregnant women, symptoms became severe immediately following parturition, suggests that reproductive steroids provide protection. It is indeed remarkable that symptoms in these patients appeared at the same time that levels of estradiol, progesterone and allopregnanolone declined postpartum. In addition to the role of immunoregulatory role of estradiol, progesterone, allopregnanolone regulates inflammatory processes by reshaping the competence of immune cells and inhibiting pro-inflammatory marks suggesting a protective role during late pregnancy in SARS-CoV-2-positive women. These observations collectively suggest that reproductive steroids may offer a treatment opportunity to improve COVID-19 symptoms in men and in post-menopausal women.

In spite of the lack of substantive data showing pregnant women are resilient to the acquisition and/or severe symptoms of COVID-19 compared with women similar in age and co-morbidities, the finding that in COVID19-positive asymptomatic pregnant women symptoms became severe immediately following parturition, suggests that reproductive steroids may be beneficial. However, in addition to pregnane steroid hormones, other hormones, including human chorionic gonadotropin (hCG), placental growth factor (PlGF), and prolactin as well as proteins produced by the placenta (e.g., leptin) have well recognized roles in immunomodulation during pregnancy and could be also involved in the worsening of COVID-19 in the postpartum period.

It is also intriguing that levels of testosterone have been implicated with immunoregulatory roles and in the context of COVID-19, high prevalence in old versus young male patients has been hypothesized to relate to lower testosterone levels in older subjects (40). Of note, not only testosterone can be aromatized to estradiol but the $5 \alpha$-reduced testosterone metabolite, $3 \alpha$-diol shows a strong estrogenic activity by binding at ERs.

Estrogens, progesterone and its metabolites are involved in multiple pharmacological effects ranging from improvement of mood disorders to analgesic properties. The anti-inflammatory action and the role in reshaping competence and increasing number of immune cells raise the hypothesis that estradiol, progesterone and allopregnanolone may alleviate COVID-19 symptoms during pregnancy.

\section{Conclusions}

Estrogens, progesterone and its physiologically-active metabolite, allopregnanolone levels increase during pregnancy and quickly drop postpartum, which is associated with increased inflammation. These hormones are involved in pleiotropic actions that range from improvement of mood disorders to anti-inflammatory properties. Estrogens and progesterone play relevant immunomodulatory functions by modulating T cell activation and inducing immune tolerance. Allopregnanolone can also protect from cognitive deficits. These actions may be significant in protecting against COVID-19 and suggest that these steroids may offer a treatment to improve COVID-19 symptoms.

\section{Figure 1}

1. Estradiol, progesterone and allopregnanolone play important physiological functions by modulating inflammatory processes and behavior. In healthy pregnant women, circulating concentrations of Estradiol progesterone and allopregnanolone rise drastically during pregnancy before decreasing dramatically following childbirth (10-12). Progesterone reaches a 100-fold higher concentration compared to baseline at 36 weeks of pregnancy. Estradiol and allopregnanolone concentrations closely parallel progesterone level increase during pregnancy and decline in the early postpartum period (11). Their concentrations increase mainly from the first to the second trimester, and then remain relatively elevated until after delivery. This hormonal progression during pregnancy is consistent with a physiological role of these reproductive steroids as hormonal modifier in pregnancy and postpartum pathophysiological processes (13). Allopregnanolone acts as a positive allosteric modulator of the inhibitory action of GABA at $\mathrm{GABA}_{\mathrm{A}}$ receptors, which has been recently associated with rapid and long-lasting improvement of postpartum depression (13). Recent reports found that COVID-19-positive pregnant patients appeared asymptomatic upon admission to the obstetrical service. However, immediately after delivery, they developed severe COVID-19 symptoms, which in some women required ICU admission (4-6). This scenario suggests that in pregnant women infected by SARS-CoV-2, reproductive steroids may provide a beneficial action. Appearance of symptoms in these patients occurred at the same time that levels 
of estradiol, progesterone and allopregnanolone decline postpartum. These hormones play an important role in regulating inflammatory processes by inhibiting pro-inflammatory markers and reshaping competence of immune cells. It is conceivable that they protect from COVID-19 symptoms during pregnancy. The hypothesis that these reproductive steroids may offer a valuable COVID-19 treatment in men and women should be verified in clinical trials.

2. Schematic representation of toll-like receptor 4 (TLR4) signaling and cross-link with allopregnanolone. In macrophages, the (TLR4-specific ligand, lipopolysaccharide (LPS), induces proinflammatory cytokines and chemokines (27). Monocytes and macrophages were studied as a model of peripheral immune signaling activation (27) showing LPS activated the TLR4 pathway with downstream activation of nuclear factor kappa-light-chain-enhancer of activated B cells (NF-kB) and increased proinflammatory mediators (MCP-1 and TNF- $\alpha$ ). Peripheral inflammation also signals to the brain with production of pro-inflammatory responses. However, TLR4 signal cascade is also expressed in neurons. Allopregnanolone acts as a positive allosteric modulator of the action of GABA at $\mathrm{GABA}_{\mathrm{A}}$ receptors and in brain mediates tonic inhibition (14-16), which has been recently, associated with rapid and long-lasting improvement of symptoms of postpartum depression in $70 \%$ of women (13). Furthermore, allopregnanolone can also dampen inflammation by acting at $\mathrm{GABA}_{\mathrm{A}}$ receptor expressed in neuroimmune cells, including macrophages, lymphocytes and glial cells. $\mathrm{GABA}_{\mathrm{A}}$ receptor forms a complex with TLR4 followed by an intracellular signal and activated (phosphorylated) transcription factors that translocate to the nucleus where they induce the synthesis of various proinflammatory markers, such as TNF- $\alpha$. However, allopregnanolone by inhibiting the GABA $\mathrm{A}_{\mathrm{A}}$ receptor-containing $\alpha 2$ subunit/TLR4 complex formation alters the intracellular signaling with a mechanism that involves blockade of TLR4/MD-2 protein interactions in macrophages that ultimately reduced production of pro-inflammatory mediators through NF- $\varkappa \mathrm{B}$-dependent or NF $\varkappa \mathrm{B}$-independent signaling pathways (27). This effect was also demonstrated in the brain. Hence, inhibition of pro-inflammatory processes both in the peripheral and neuroimmune signaling, which can be initiated by allopregnanolone binding at $\mathrm{GABA}_{\mathrm{A}}$ receptor in monocytes, macrophages and brain cells, underscores the anti-inflammatory effects of allopregnanolone and underlies its novel function to regulate periphery and brain immune responses.

3. Schematic representation of immunomodulatory effects of progesterone. In the periphery, progesterone directly regulates $\mathrm{T}$ cell activation and differentiation by: 1) $\mathrm{T}$ cell activation; 2) Inducing tolerance; 3) Cytotoxicity; and 4) Inducing tolerance at maternal fetal interface. Modified from (30).

\section{References}

1. Coronavirus COVID-19 global cases by the Center for Systems Science and Engineering. Last accessed 3/29/20 (https://www.arcgis.com/apps/opsdashboard/index.html\#/bda7594740fd40299423467b48e9ecf6).

2. Peckham h, de Gruijter N, Raine C, Radziszewska A, Ciurtin C, Wedderburn LR, Rosser EC, Deakin C, Webb K. Sex-bias in COVID-19: a meta-analysis and review of sex differences in disease and immunity.

Infectious Disease 2020. 10.21203/rs.3.rs-23651/v1

3. https://www.worldometers.info/coronavirus/coronavirus-symptoms/

4. Breslin N, Baptistec C, Miller R, Fuchs, K, Goffman D, Gyamfi-Bannerman C, D'Alton M. COVID-19 in pregnancy: early lessons. American Journal of Obstetrics and Gynecology 2020

5. Breslin N, Baptiste C, Gyamfi-Bannerman C, Miller R, Martinez R, Bernstein K, Ring L, Landau R, Purisch S, Friedman AM, Fuchs K, Sutton D, Andrikopoulou M, Rupley D, Sheen JJ, Aubey J, Zork N, Moroz L, Mourad M, Wapner R, Simpson LL, D'Alton ME, and Goffman D. COVID-19 infection among asymptomatic and symptomatic pregnant women: Two weeks of confirmed presentations to an affiliated pair of New York City hospitals. American Journal of Obstetrics $\& 3$ Gynecology MFM. 9 April 2020, 100118

6. Wu C, Yang W, Wu X, Zhang T, Zhao Y, Ren W, Xia J. Clinical manifestation and laboratory characteristics of SARS-CoV-2 infection in pregnant women. Virol Sin . 2020 Apr 20. doi: 10.1007/s12250-020-00227-0

7. Yu N, Li W, Kang Q, Xiong Z, Wang S, Lin X, Liu Y, Xiao J, Liu H, Deng D, Chen S, Zeng W, Feng L, 
Wu J. Clinical features and obstetric and neonatal outcomes of pregnant patients with COVID-19 in Wuhan, China: a retrospective, single-centre, descriptive study. Lancet Infect Dis. 2020 May;20(5):559-564. doi: 10.1016/S1473-3099(20)30176-6.

8. Chen H, Guo J, Wang C, et al. Clinical characteristics and intrauterine vertical transmission potential of COVID-19 infection in nine pregnant women: a retrospective review of medical records. Lancet. 2020:395;809-815.

9. Sutton D, Fuchs K, D'Alton M, Goffman D. Universal Screening for SARS-CoV-2 in Women Admitted for Delivery. New England Journal of Medicine. DOI: 10.1056/NEJMc2009316

10. Luisi F., Petraglia, Benedetto C, Nappi RE, Bernardi F, Fadalti M, Reis FM, Luisi M, and Genazzani AR. Serum Allopregnanolone Levels in Pregnant Women: Changes during Pregnancy, at Delivery, and in Hypertensive Patients J Clin Endocrinol Metab. 2000 Jul;85(7):2429-33.

11. Kurt D. Pennell, Mark A. Woodin, Page B. Pennell. Quantification of neurosteroids during pregnancy using selective ion monitoring mass spectrometry. Steroids 95 (2015) 24-31

12. Deligiannidis KM, Kroll-Desrosiers AR, Mo S, Nguyen HP, Svenson A, Jaitly N, Hall JE, Barton BA, Rothschild AJ, Shaffer SA. Peripartum neuroactive steroid and $\gamma$-aminobutyric acid profiles in women at-risk for postpartum depression Psychoneuroendocrinology. 2016 Aug; 70: 98-107. doi: 10.1016/j.psyneuen.2016.05.010

13. Meltzer-Brody SE, Kanes SJ. Allopregnanolone in postpartum depression: Role in pathophysiology and treatment. Neurobiology of stress. https://doi.org/10.1016/j.ynstr.2020.1002129.

14. Paul SM, Pinna G, Guidotti A. "Allopregnanolone: From Molecular Pathophysiology to Therapeutics A Historical Perspective. Neurobiology of Stress. 2020 DOI: 10.1016/j.ynstr.2020.100215

15. Locci A and Pinna G. Neurosteroid biosynthesis downregulation and changes in GABAA receptor subunit composition: A biomarker axis in stress-induced cognitive and emotional impairment. British Journal of Pharmacology 174:3226-3241

16. Pinna G, Uzunova V, Matsumoto K, Puia G, Mienville JM, Costa E and Guidotti A: Brain allopregnanolone regulates the potency of the $\mathrm{GABA}_{\mathrm{A}}$ receptor agonist muscimol. Neuropharmacology 39:440-448. 2000 .

17. Maguire JL, Stell BM, Mody I. Ovarian cycle-linked changes in GABAA receptors mediating tonic inhibition alter seizure susceptibility and anxiety. Nat Neurosci 2005; 8: 797-804.

18. Bloch M, Schmidt PJ, Danaceau M, Murphy J, Nieman L, Rubinow DR. Effects of gonadal steroids in women with a history of postpartum depression. Am J Psychiatry. 2000 Jun;157(6):924-30.

19. Wong CG, Bottiglieri T, Snead OC 3rd (2003) GABA, gamma-hydroxybutyric acid, and neurological disease. Ann Neurol 54(Suppl 6):S3-S12.

20. Clarkson AN, Sutherland BA, Appleton I (2005) The biology and pathology of hypoxiaischemia: An update. Arch Immunol Ther Exp (Warsz) 53:213-225.

21. Clarkson AN, et al. (2005) Clomethiazole: Mechanisms underlying lasting neuroprotection following hypoxia-ischemia. FASEB J 19:1036-1038.

22. Reyes-Garcia MG, Hernandez-Hernandez F, Hernandez-Tellez B, Garcia-Tamayo F (2007) GABA (A) receptor subunits RNA expression in mice peritoneal macrophages modulate their IL-6/IL-12 production. J Neuroimmunol 188:64-68.

23. Tian J, et al. (2004) Gamma-aminobutyric acid inhibits T cell autoimmunity and the development of inflammatory responses in a mouse type 1 diabetes model. J Immunol 173:5298-5304. 
24. Alam S, Laughton DL, Walding A, Wolstenholme AJ (2006) Human peripheral blood mononuclear cells express GABAA receptor subunits. Mol Immunol 43:1432-1442

25. Bergeret M, et al. (1998) GABA modulates cytotoxicity of immunocompetent cells expressing GABAA receptor subunits. Biomed Pharmacother 52:214-219.

26. Bhat R, Axtell R, Mitra A, Miranda M, Lock C, Tsie RW, and Stein L. Inhibitory role for GABA in autoimmune inflammation. Proc Natl Acad Sci U S A. 2010 Feb 9;107(6):2580-5. doi: 10.1073/pnas.0915139107

27. Suresh K. Mendu, Amol Bhandage, Zhe Jin, Bryndis Birnir. Different Subtypes of GABA-A Receptors Are Expressed in Human, Mouse and Rat T Lymphocytes. https://doi.org/10.1371/journal.pone.0042959

28. Boero G, Porcu P, and A. Leslie Morrow. Pleiotropic actions of allopregnanolone underlie therapeutic benefits in stress-related disease Neurobiol Stress. 2020 May; 12: 100203. Published online 2019 Nov 27. doi: $10.1016 /$ j.ynstr.2019.100203

29. Balan I, Beattie MC, O'Buckley TK, Aurelian L, and Morrow AL. Endogenous Neurosteroid $(3 \alpha, 5 \alpha) 3$-Hydroxypregnan-20-one Inhibits Toll-like-4 Receptor Activation and Pro-inflammatory Signaling in Macrophages and Brain Sci Rep. 2019; 9: 1220. Published online 2019 Feb 4. doi: 10.1038/s41598-018$37409-6$

30. Nishel M. Shah, Pei F. Lai, Nesrina Imami, and Mark R. Johnson. Progesterone-Related Immune Modulation of Pregnancy and Labor Front Endocrinol (Lausanne). 2019; 10: 198. Published online 2019 Mar 29. doi: 10.3389/fendo.2019.00198

31. Hall, O., Klein, S. Progesterone-based compounds affect immune responses and susceptibility to infections at diverse mucosal sites. Mucosal Immunol 10, 1097-1107 (2017). https://doi.org/10.1038/mi.2017.35

32. Vermillion MS, Nelson A, Vom Steeg L, Loube J, Mitzner W, Klein SL. Pregnancy preserves pulmonary function following influenza virus infection in C57BL/6 mice. Am J Physiol Lung Cell Mol Physiol. 2018 Oct 1;315(4):L517-L525. doi: 10.1152/ajplung.00066.2018. Epub 2018 May 31.

33. Goldfarb I , Berk T, Phillippe M. Pregnancy levels of estradiol and progesterone modulate lung inflammation. AJO\&G DOI:https://doi.org/10.1016/j.ajog.2014.10.525

34. Maglione A, Rolla S, Mercanti SF, Cutrupi S, Clerico M. The Adaptive Immune System in Multiple Sclerosis: An Estrogen-Mediated Point of View. Cells. 2019 Oct 19;8(10). pii: E1280. doi: 10.3390/cells8101280.

35. Ghisletti S, Meda C, Maggi A, and Vegeto E. 17-Estradiol Inhibits Inflammatory Gene Expression by Controlling NF-B Intracellular Localization MOLECULAR AND CELLULAR BIOLOGY, Apr. 2005, p. 2957-2968 Vol. 25, No. 8 0270-7306/05/\$08.000 doi:10.1128/MCB.25.8.2957-2968.2005

36. Gubbels Bupp, M.R.; Potluri, T.; Fink, A.L.; Klein, S.L. The Confluence of Sex Hormones and Aging on Immunity. Front. Immunol. 2018, 9, 1269

37. Paris J, Liere P, Kim S, Mahdi F et al., Pregnane steroidogenesis is altered by HIV-1 Tat and morphine: Physiological allopregnanolone is protective against neurotoxic and psychomotor effects https://doi.org/10.1016/j.ynstr.2020.100211

38. Maingat FG, Polyak MJ, Paul AM, Vivithanaporn P, Noorbakhsh F, Ahboucha S, Baker GB, Pearson K, Power C. Neurosteroid-mediated regulation of brain innate immunity in HIV/AIDS: DHEA-S suppresses neurovirulence. Faseb. J., 27 (2013), pp. 725-737, 10.1096/fj.12-215079

39. Butler SA, Cole LA. Evidence for, and Associated Risks with, the Human Chorionic Gonadotropin Supplemented Diet. J Diet Suppl. 2016 Nov;13(6):694-9. doi: 10.3109/19390211.2016.1156208. Epub 2016 Mar 24. Review.

40. Pozzillia $\mathrm{P}$ and Lenzib A. Testosterone, a key hormone in the context of COVID-19 pandemic Metabolism. 2020 Apr 27 : 154252. doi: 10.1016/j.metabol.2020.154252 [Epub ahead of print] 


\section{Hosted file}

Figure 1.pptx available at https://authorea.com/users/320698/articles/450155-progesteroneand-estradiol-may-be-beneficial-for-the-treatment-of-covid-19-hypothesis-paper 